\title{
Inference for bounded parameters
}

\author{
D.A.S. Fraser and N. Reid \\ Department of Statistics, University of Toronto \\ Toronto Canada M5S 3G3 \\ A. Wong \\ Department of Mathematics and Statistics, York University \\ Toronto Canada M3J 1P3
}

November 5, 2018

\begin{abstract}
The estimation of signal frequency count in the presence of background noise has had much discussion in the recent physics literature, and Mandelkern [1] brings the central issues to the statistical community, leading in turn to extensive discussion by statisticians. The primary focus however in [1] and the accompanying discussion is on the construction of a confidence interval. We argue that the likelihood function and $p$-value function provide a comprehensive presentation of the information available from the model and the data. This is illustrated for Gaussian and Poisson models with lower bounds for the mean parameter.
\end{abstract}

\section{INTRODUCTION}

Mandelkern [1] brings to the statistical community a seemingly simple statistical problem that arises in high energy physics; see for example, [2], [3]. The statistical model is quite elementary but the related inference problem has substantial scientific presence: as Pekka Sinervo, a coauthor of Abe et al. [2], [3] expresses, "High energy physicists have struggled with Bayesian and frequentist perspectives, with delays of several years in certain experimental programmes hanging in the balance". 
The problem discussed in [1] can be expressed simply. A variable $y$ follows a distribution with mean $\theta=b+\mu$, where $b>0$ is known, the shape of the distribution is known and the parameter $\mu \geq 0$. The goal is to extract the evidence concerning the parameter $\mu$, and in particular present the evidence on whether $\mu$ is zero or is greater than zero. In the physics setting $y$ is often a count and is viewed as the sum of a count of $y_{1}$ background events and a count of $y_{2}$ events from a possible signal. In [2] and [3], the signal records the presence of a possible top quark and the data come from the collider detector at Fermilab. The background count $y_{1}$ is modelled as Poisson $(b)$ and the count from the possible signal as Poisson $(\mu)$. Following Mandelkern [1] we write $y \sim \operatorname{Poisson}(b+\mu)$ and let $\theta=b+\mu$ be the Poisson mean with the restriction $\theta \geq b$. There are additional aspects: for example the data are obtained as subsets of more complex counts, the background mean count $b$ is estimated and so on, but we concentrate on the simpler problem here. We do however illustrate how the general case with $b$ estimated from antecedent Poisson counts can be treated within the general theory.

The Poisson case involves a discrete distribution and this introduces some minor complications that that are best treated separately from the essential inference aspects. Accordingly we include a discussion of the continuous case and for simplicity consider the normal distribution for $y$ with mean $\theta=b+\mu$ and known standard deviation.

Much statistical literature and most of the physics proposals cited by Mandelkern [1] are concerned with the construction of confidence bands for $\theta$ at some prescribed level of confidence. It is our view that this leads to procedures that are essentially decision-theoretic: we "accept" parameter values within the confidence interval and "reject" parameter values outside the interval; a $1 / 0$ presentation. This accept/reject theory evolved from Neyman and Pearson [4], later generalized as decision theory by Wald [5]. The decision theoretic approach dominated statistical theory until the mid 1950's, when Savage [6] promoted the personalistic Bayesian approach and Fisher [7] recommended an inference approach. Both these approaches make essential use of the likelihood function: the Bayesian approach combines this with prior information, and the inference approach emphasizes the use the likelihood function and the observed significance or $p$-value function. The $p$ value function is constructed using the model and observed data, as we shall describe in more detail below. One difficulty with the confidence interval approach arises from the presence of the lower bound $b$ for the parameter space; if $y$ is small then the confidence interval can be partly or completely 
outside the permissible range $[b, \infty)$ for the parameter, making apparent nonsense of an assertion of 95\% confidence. Various proposals have been put forward to modify the confidence approach to overcome such difficulties; the most prominent being the unified approach of Feldman and Cousins [8]. These proposals seek an algorithm for placing a $1 / 0$ valuation on possible parameter values, in the framework of a prescribed confidence level. By contrast the $p$-value function promoted here provides essential evidence from the data concerning the value of the parameter; for some background see Fraser [9].

The discussants of Mandelkern [1] also focus on the confidence interval approach. An exception is Gleser [10], who suggests the use of the "likelihood function as a measure of evidence about the parameters of the model used to describe the data"; and Mandelkern [11] in his rejoinder concurs: "it may be most appropriate to, at least in ambiguous cases, give up the notion of characterizing experimental uncertainty with a confidence interval ... and to present the likelihood function for this purpose." But also Abe et al [3] report the $p$-value for the parameter value $\mu=0$; the $p$-value function extends this to all possible values of the parameter. The approach here recommends the joint presentation of the likelihood function and the $p$-value function as the evidence from the data concerning the parameter.

In Section 2 we record some discussion of the unified approach and its variants, and also record various anomalies associated with their use.

In Section 3 we expand on Mandelkern's comment and discuss what we call an inferential approach. This records the observed likelihood function and the observed $p$-value function. We feel that these present the full statistical evidence concerning the parameter, and in turn allow appropriate individual judgments to be made concerning the parameter. An experiment reported in [3] is analysed using the Poisson model with background, first for known background and then allowing Poisson variation in the background.

\section{The unified approach and variants}

The construction of a confidence interval is often based on the theory of optimal testing, and this can lead to rather anomalous behavior. An optimality criterion typically involves averaging over the sample space, and in many situations there are what Fisher [7, 12] called 'recognizable subsets' of the sample space, subsets that appropriately partition the sample space. 
In this setting the use of overall optimality can mean that intervals are constructed which effectively trade performance in a single instance for average performance in a series of instances, most of which may have recognizably different features. In extreme cases this can give a confidence interval that is empty or a confidence interval that is the full range for the parameter: in such cases the overt confidence is clearly zero or $100 \%$ in contradiction to the prescribed or targetted confidence. For some recent discussions with examples, see Fraser [9] where the optimality criteria are shown to lead to decisions that are contrary to the available evidence; see also Cox [13] on the general appropriateness of optimality criteria.

The conventional intervals applied to examples with a bounded parameter space also can lead to anomalous confidence intervals. Thus an optimum confidence interval derived for the unrestricted case may well lap into the inappropriate region $\theta<b$, this being the key issue in the Poisson case and mentioned for the continuous case in Mandelkern [1].

Various proposed modifications to the typical central confidence interval are discussed in Mandelkern [1]. Assume we have a scalar variable $y$ with a continuous density $f(y ; \theta)$ and with a distribution function $F(y ; \theta)$ that is stochastically increasing in $\theta$. Denote by $y_{L}(\theta)$ and $y_{U}(\theta)$ the $\gamma$ and $95 \%+\gamma$ quantiles of $F(y ; \theta)$; these form a $95 \%$ confidence interval. Now let $\gamma=\gamma(\theta)$ vary with $\theta$ but be restricted to the interval $(0,5 \%)$. The confidence belt in the $y \times \theta$-space is the set union of the acceptance regions $\left(y_{L}(\theta), y_{U}(\theta)\right) \times\{\theta\}$; and the $y$-section of the two dimensional confidence belt is a $95 \%$ confidence region and under moderate regularity will have the form $\left(\theta_{L}(y), \theta_{U}(y)\right)$. A reasonable objective is to have these sets stay within the acceptable range $[b, \infty)$ by some natural-seeming choice of the adjustment function $\gamma(\theta)$.

The likelihood ratio is used as one basis for deciding which points are to go into the acceptance interval $\left(y_{L}(\theta), y_{U}(\theta)\right)$ and thus for determining $\gamma(\theta)$. Then to form the acceptance interval the points are ordered from the largest using the ratio

$$
R=\frac{L(\theta ; y)}{L(\tilde{\theta} ; y)}
$$

where $L(\theta ; y)=f(y ; \theta)$ and $\tilde{\theta}=\tilde{\theta}(y)$ is a reference parameter value to be used with $y$. The Unified Approach of Feldman \& Cousins [8] takes $\tilde{\theta}=\hat{\theta}(y)$ to be the maximum likelihood estimate of $\theta$ under the restriction $\theta \geq b$; for example in the $\operatorname{Normal}(\theta, 1)$ case, we have $\hat{\theta}=\max (b, y)$. The New Ordering approach of Giunti [14] takes $\tilde{\theta}$ to be a Bayesian expected value 
for $\theta$. Using a somewhat different starting point Mandelkern \& Schultz [15] obtain likelihood from the distribution of $\hat{\theta}(y)$, which is a marginalisation from the distribution of $y$ itself. For the normal case this $\hat{\theta}$ does not depend on $y$ for $y<b$ and not surprisingly the confidence intervals obtained by this approach are found not to depend on $y$ for $y<b$; the resulting intervals had been considered earlier by Ciampolillo [16]. The use of these optimizing or ordering criteria can have rather strange effects. For, as noted, the criteria involve shifting the distribution bound to the left for low parameter values so that the $2.5 \%$ tail probabilities on the left and the right are changed to have less on the left and more on the right; this has the effect for small data values of shifting the confidence intervals to the right, away from the excluded parameter value range. The disturbing result however is that the lower confidence bound is no longer a $2.5 \%$ bound but something larger and perhaps undefined. And the upper confidence bound is no longer a $97.5 \%$ bound but something larger and perhaps undefined. Thus the individual bounds of the confidence interval do not have the direct statistical meaning that one would reasonably impute to them; this is particularly serious and disturbing in a context where the lower bound is directly addressing the issue of whether or not $\mu$ is equal to zero. These approaches seem to seek a single construction that combines the merits of one-sided and two-sided confidence intervals. In a sense this is treating both $b$ and $\theta$ as parameters and having the same construction provide conclusions about both of them. The inferential approach of the next section emphasizes the evidence in the data about the single parameter $\theta$, with $b$ fixed. The extension to the case of estimated background illustrated in Section 4 emphasizes the evidence in the data about $\theta$, in the presence of a nuisance parameter.

For the Poisson problem described in the introduction, Roe \& Woodroofe [17] propose the use of certain conditional probabilities as the basis for the confidence belt construction following Feldman \& Cousins [8]. Such conditioning had been proposed earlier for upper limits by Zech [18]. Roe and Woodroofe [17] recommended the use of the conditional distribution of $y$ given $y_{1} \leq y^{0}$, say $g(y ; \mu)=f\left(y \mid y_{1} \leq y^{0} ; \mu\right)$ as recorded (4) in Mandelkern [1]. But the variable $y_{1}$ is not an observable variable and hence not ancillary in the usual sense, and the proposed conditioning does not generate a partition of the sample space. This was noted in Woodroofe \& Wang [19] and in Cousins [20], and a Bayesian approach was proposed in Roe and Woodroofe [21]. Thus the nominal conditional distribution does not satisfy the standard conditions for validity in describing conditional frequencies given observed 
information. Also not surprisingly, as noted by Mandelkern [1] and Cousins [20], there is a related undercoverage which can be severe for the nominal confidence intervals constructed.

\section{The statistical evidence: the likelihood and $p$-value functions}

Consider first a sample $\underline{y}=\left(y_{1}, \ldots, y_{n}\right)$ from the Normal $\left(\theta, \sigma_{0}^{2}\right)$ distribution with $\sigma_{0}^{2}$ known. The likelihood function is proportional to the density for the sample mean at the observed value $\bar{y}^{0}$, and is examined as a function of the unknown $\theta$ :

$$
L(\theta)=c \phi\left(n^{1 / 2}\left(\bar{y}^{0}-\theta\right) / \sigma_{0}\right),
$$

where $\phi$ is the standard normal density. The $p$-value function is the probability that the sample mean is less than or equal to the observed $\bar{y}^{0}$ :

$$
p(\theta)=\Phi\left(n^{1 / 2}\left(\bar{y}^{0}-\theta\right) / \sigma_{0}\right)
$$

where $\Phi$ is the standard normal distribution function. The $p$-value function uses the known sampling distribution of $\bar{y}$, and records the percentile position of the observed data in the distribution having parameter value $\theta$. The more conventional interpretation of the $p$-value as "the probability of observing a result as or more extreme, under the model" is obtained as 1 minus the $p$-value function when the data is in the right tail of the distribution. Twotailed $p$-values can also be obtained if desired. As a function of $\bar{y}, p(\theta)$ is uniformly distributed on $(0,1)$ under the assumed model. This "repeated sampling" property of the $p$-value is the analogue of coverage of a confidence interval.

This discussion extends directly to any location model $f(y-\theta)$ for $y$. The likelihood function is

$$
L(\theta)=L\left(\theta ; y_{1}^{0}, \ldots, y_{n}^{0}\right)=c\left(\underline{y}^{0}\right) \prod_{i=1}^{n} f\left(y_{i}^{0}-\theta\right) .
$$

And the $p$-value function, using the sampling distribution of $\bar{y}$ conditional on the observed sample configuration $a^{0}=\left(y_{1}^{0}-\bar{y}^{0}, \ldots, y_{n}^{0}-\bar{y}^{0}\right)$, is

$$
p(\theta)=\int_{-\infty}^{y^{0}} f\left(\bar{y} \mid a^{0}\right) d \bar{y}
$$


in the special Gaussian case $\bar{y}$ is independent of $a$. This raises essentially no new problems beyond the computation of the integral. For this location model it can be shown that the $p$-value function is identical to the integral of the likelihood function, so that

$$
p(\theta)=\int_{\theta}^{\infty} L(\nu) d \nu / \int_{-\infty}^{\infty} L(\nu) d \nu
$$

thus the $p$-value function is identical to the posterior survivor function (one minus the posterior distribution function) using the flat prior $\pi(\theta) d \theta=d \theta$.

The location form of the model provides a procedure for simplifying the data vector $\left(y_{1}, \ldots, y_{n}\right)$ to a scalar summary, $\bar{y}$, by conditioning. As the distribution of the sample configuration is free of $\theta$, no information is lost by this conditioning. The use of $\bar{y}$ as the one-dimensional variable is not essential; the same result is obtained using the maximum likelihood estimate, or in fact any location respecting estimator of $\theta$, together with a notational change in the expression for $a$. In the methods for more general models this argument is applied using approximate conditioning and reexpression of the parameter to location form.

We now return to the Poisson $(\theta)$ with $\theta=b+\mu$ where $b$ is known and $\mu \geq 0$. The Poisson case is simpler, in that the model specifies a onedimensional variable, $y$, and a one-dimensional parameter $\theta$, so no dimension reduction is needed. The likelihood function from an observed count $y^{0}$ is

$$
L(\theta)=c \theta^{y^{0}} e^{-\theta}
$$

where $\theta=b+\mu$. This can be plotted as a function of $\mu$ for $\mu$ in $(-b, \infty)$ : for $\mu$ in $[0, \infty)$ it describes the probability at the observed data point under the assumed model; for $\mu$ in $[-b, 0)$ it serves as a diagnostic concerning $b$, suggesting that either the model or the computation of $b$ is not correct. The $p$-value function at $y^{0}$ is given by the interval

$$
p(\theta)=\left(F^{-}\left(y^{0} ; \theta\right), F\left(y^{0} ; \theta\right)\right)
$$

of numerical values, where $F(y ; \theta)$ is the $\operatorname{Poisson}(\theta)$ distribution function and $F^{-}(y ; \theta)$ is the probability up to, but not including, $y$ and is given by $F(y-1 ; \theta)$. We use an interval of $p$-values in accord with the discreteness in the problem; a compromise is to plot the so-called mid p-value, which is $F^{-}\left(y^{0} ; \theta\right)+(1 / 2) f\left(y^{0} ; \theta\right)$. In our approach an observed $y^{0}$ leads to a continuum of numerical $p$-values for each $\theta$ being assessed. This proposal acknowledges the discreteness explicitly and yet does maintain the repeated sampling 
property of the $p$-value function, that it have a uniform distribution on $(0,1)$. Other aspects of the discreteness problem are addressed in Brown et al. [22] and Baker [23].

As a simple example consider $b=2$ with data $y^{0}=3$. The likelihood and $p$-value functions are recorded in Figure 1. The likelihood for $\mu$ is easily understood, and particularly useful when combining data. The interpretation of a $p$-value for given data value is exactly analogous to the percentile score on, for example, a standardized test: it expresses the percentile position of the data point relative to the parameter. For the null condition $\theta=2$ or $\mu=0$ the $p$-value interval for the data $y^{0}=3$ is $(0.6770 .857)$; a fairly central and broad range.

If $y^{0}=0$, and $b>0$, then $p(\mu)=(0, \exp \{-(b+\mu)\})$. This emphasizes the lack of information in the data about $\mu$, and this lack is most striking when $\mu=0$ and $b$ is very small. For larger $b$, the observed value of 0 will be further in the left tail of the $\mu=0$ distribution.

In Abe et al. [3] after preliminary simplification from their Table 1 we have $b=6.7$ with $y^{0}=27$. The likelihood function and $p$-value functions are plotted in Figure 2. For the null condition $\theta=6.7$ or $\mu=0$ the data is in the extreme right tail and the upper and lower $p$-values are essentially 1 . The actual values are $\left(1-3 \times 10^{-8}, 1-10^{-8}\right)$ thus offering very strong evidence that $\mu>0$.

Figure 3 shows the corresponding likelihood and $p$-value plot for a Gaussian model, with $\mu=b+\theta$, where $n=1, \sigma=0.5$ and $y=1.8705$ with $b$ taken to be 1.4142 .

The Gaussian case is not as far removed from the Poisson as might be thought at first. If $y \sim \operatorname{Poisson}(\theta)$, then $\sqrt{ } y$ is approximately distributed as Gaussian with mean $\sqrt{ } \theta$ and standard deviation $1 / 2$, at least for large $\theta$. For comparison with the first example and Figure 1 , the $p$-value interval for testing $\mu=0$ computed using the normal approximation with continuity correction, i.e. evaluated at $\sqrt{ }\left(y^{0} \pm 0.5\right)$, is $(0.631,0.819)$.

It is possible to use recently developed work in likelihood asymptotics in the Poisson model with estimated background. We suppose that the background mean count $\beta$ is an unknown parameter estimated by $b$. To reflect the precision in this estimate, we write

$$
b=y_{1} / k
$$

where $y_{1}$ follows a Poisson distribution with mean $k \beta$ and hence variance $k \beta$. A value for the standard error of $b$, say $\sigma_{b}$ determines a value for $k$ as 
Figure 1: The likelihood function (top) and $p$-value function (bottom) for the Poisson model, with $b=2$ and $y^{0}=3$. For $\mu=0$ the $p$-value interval is $(0.677,0.857)$.
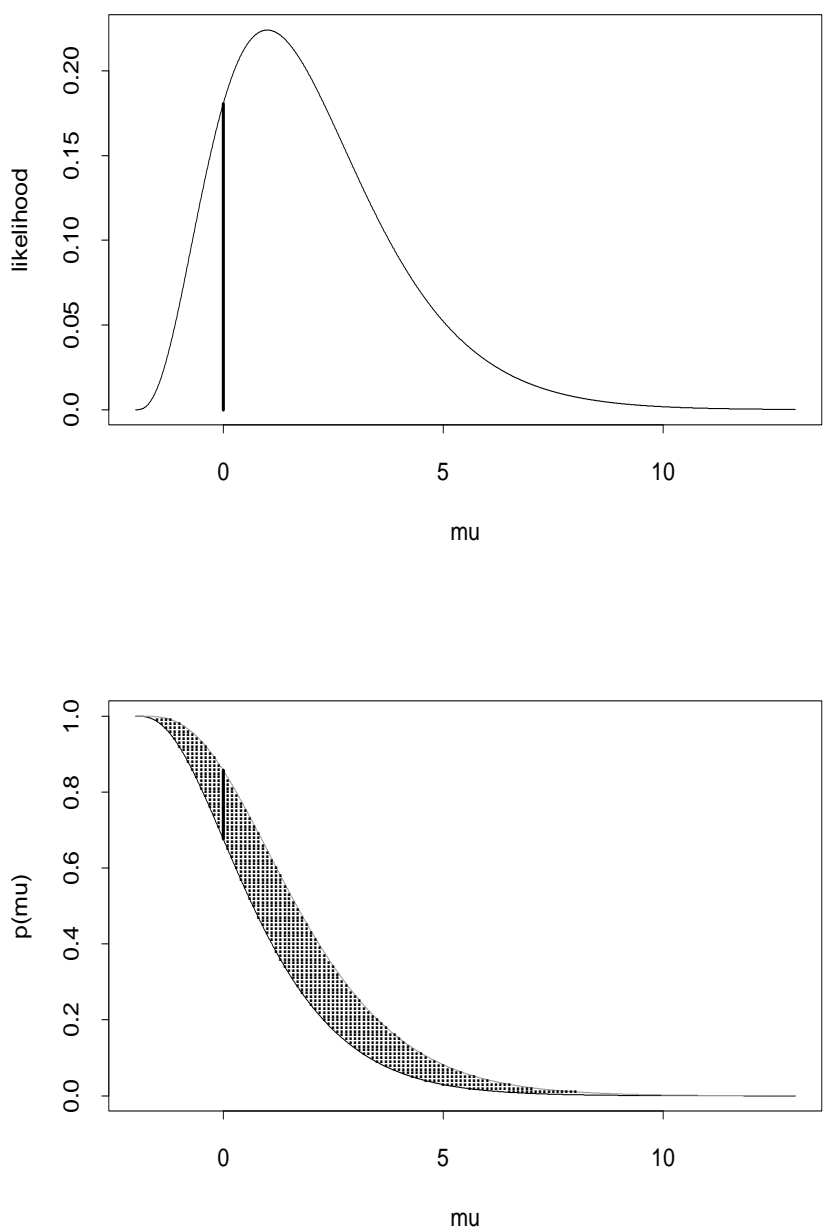
Figure 2: The likelihood function (top) and $p$-value function (bottom) for the Poisson model, with $b=6.7$ and $y^{0}=27$. For $\mu=0$ the upper and lower $p$-values are essentially 1.
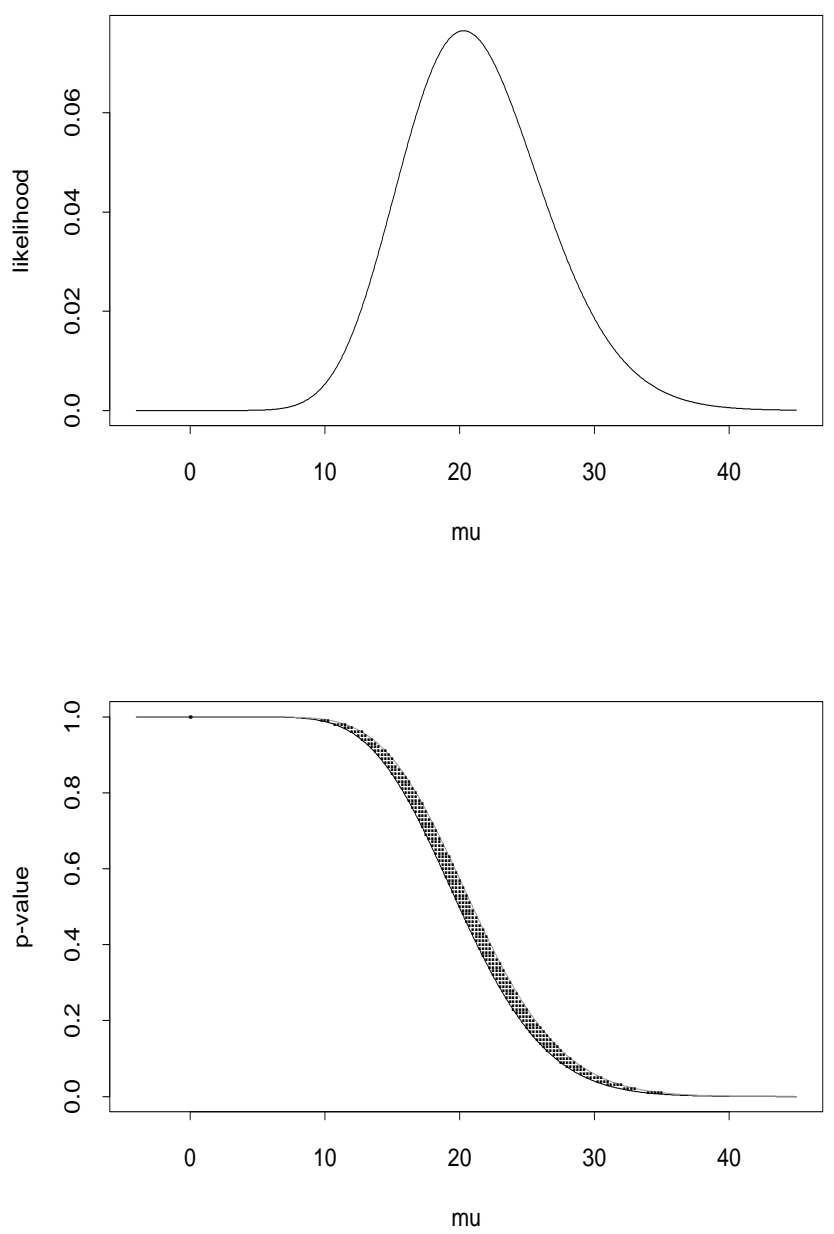
Figure 3: The likelihood function (top) and $p$-value function (bottom) for the normal approximation to the Poisson model, after square root transformation with data as in Figure 1.
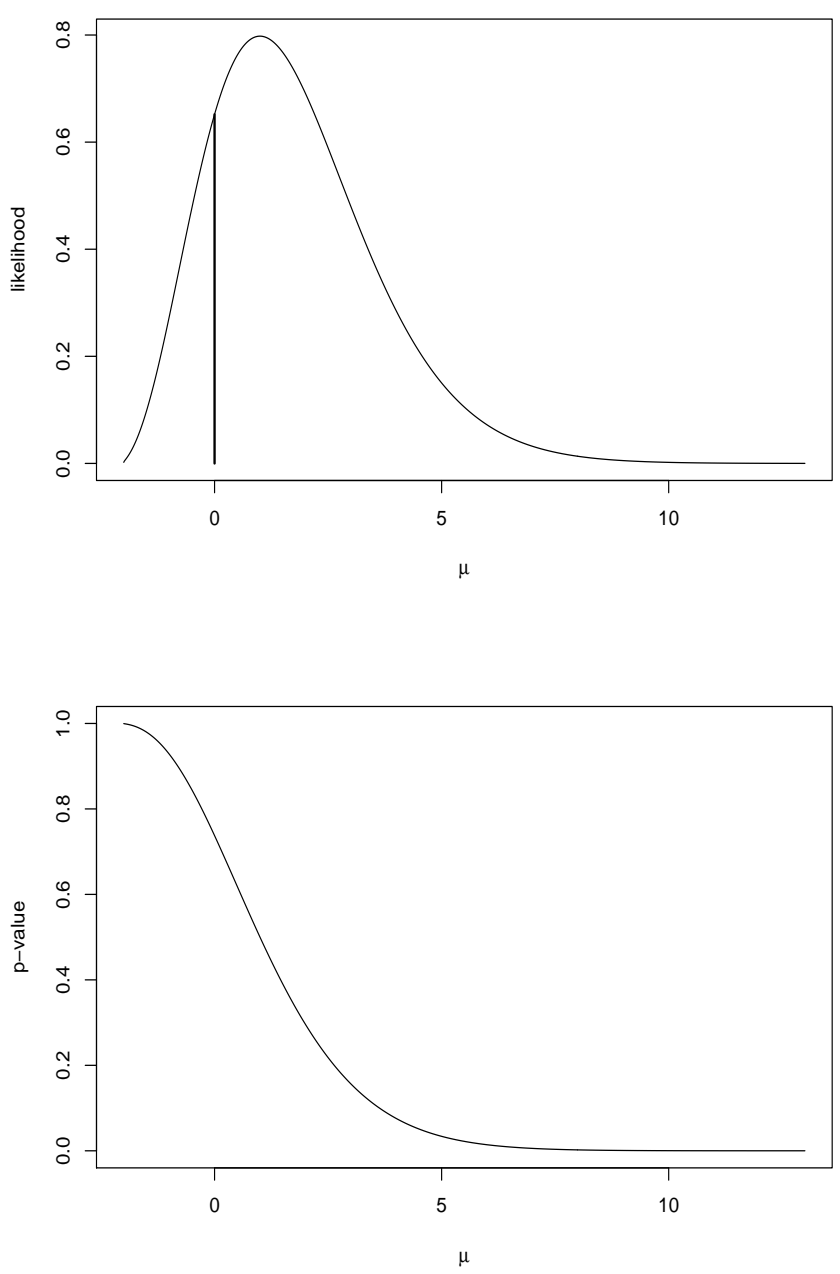
$k=b / \sigma_{b}^{2}$. In [3] the estimated standard error from Table II is 2.1, with

$b=6.7$. The resulting $p$-value function is plotted in Figure 4, where it is compared with the mid $p$-value function assuming the background is known. The value of the new $p$-value function at $\mu=0$ is $1-2.6 \times 10^{-5}$.

\section{Discussion}

The $p$-value function, evaluated at a particular value $\theta_{0}$, gives the percentile position of the observed data relative to the model with that parameter value $\theta_{0}$. Our view is that the $p$-value provides the key scientific evidence in the data relative to the assumed model. In contrast a fixed level confidence approach provides a much more limited statement that the parameter is or is not contained in a given interval. An improvement to the confidence approach would be the reporting of confidence limits at a continuum of confidence levels, which is mathematically close to the $p$-value function approach. One can use the $p$-value function to construct a confidence interval at level $1-\alpha$, by finding the parameter values for which the $p$-value equals, say, $1-\alpha / 2$ and $\alpha / 2$. However our definition of the $p$-value function is intrinsically one-sided, as seems more appropriate for the physical context of detecting a signal.

It is important to know how the inferential approach promoted here generalizes to more complex models. Most realistic models will have a parameter $\theta$ of dimension $d$, say. For this setting we might be interested in a scalar component $\psi(\theta)$ and could then want the $p$-value function for $\psi$. If more than one component of $\theta$ is of particular interest each could be examined in turn. The essential simplification available for this setting from recently developed likelihood theory is that to a high order of approximation there is a conditional model that behaves like a location model for $\psi$ with a related scalar variable that measures this parameter. Approximations to the corresponding observed likelihood function are given in Fraser [24] and approximations to the $p$-value function are given in Fraser, Reid and $\mathrm{Wu}$ [25]. These evolved from a closely related approach based on ancillarity due to Barndorff-Nielsen which is summarized in Barndorff-Nielsen and Cox [26]. The approach as described in [25] requires that $y$ follow a continuous distribution in general models. Work in progress with A.C. Davison extends this approach to the discrete setting, and this work was used to derive the results summarized in Figure 4.

The statistical literature summarizing higher order likelihood asymptotics 
Figure 4: The $p$-value function using the third order approximation developed from [25], allowing for estimation errors in the background signal, compared with the mid $p$-value assuming the background is known.

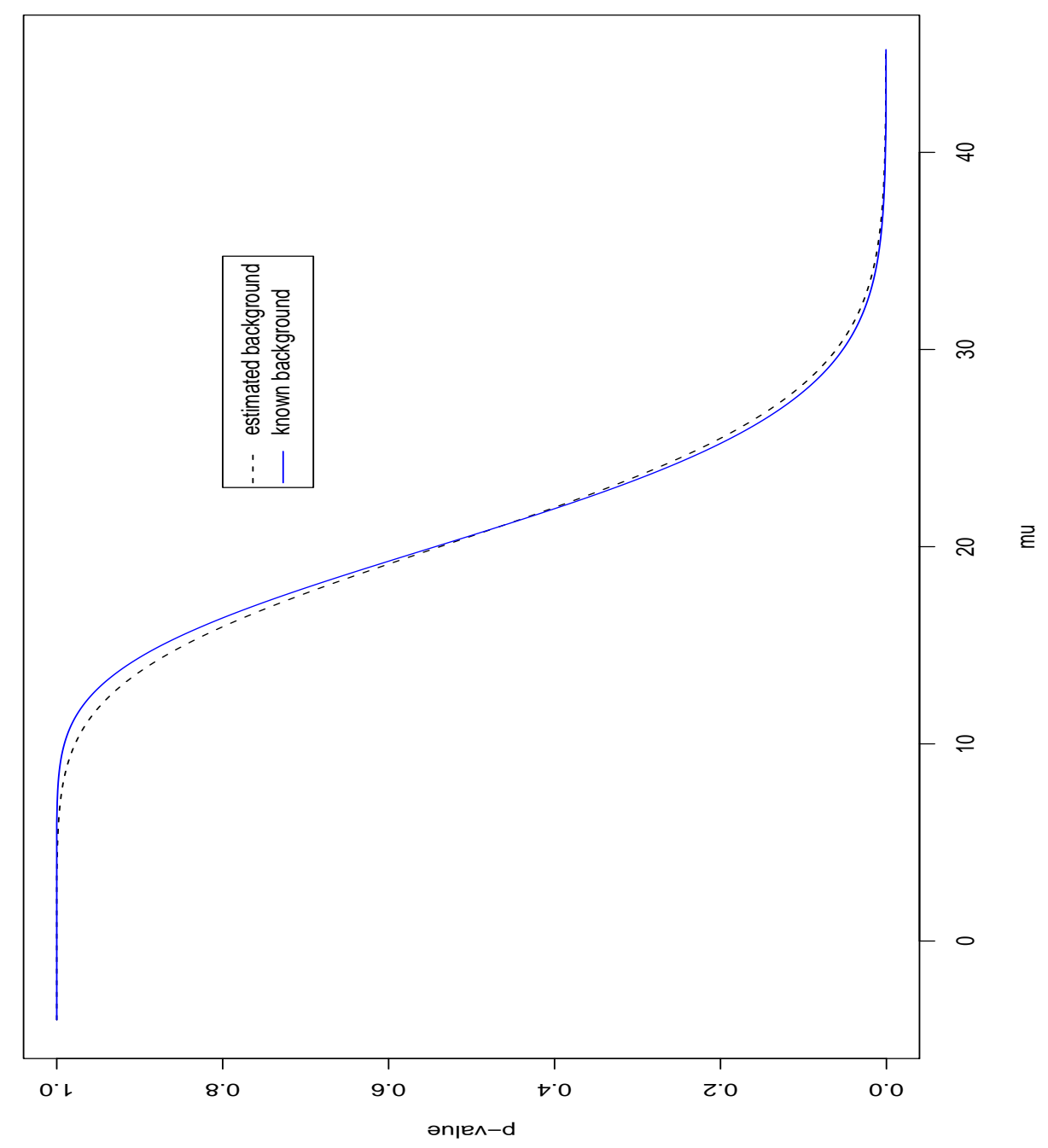


is still fairly specialized, but some review or book length treatments are available in Reid [27], Severini [28], Skovgaard [29] and Barndorff-Nielsen and Cox [26].

\section{Acknowledgements}

The authors wish to thank the referees for many very helpful comments that assisted in the revision of an earlier version. The research was partially supported by the Natural Sciences and Engineering Research Council of Canada. 
[1] M. Mandelkern, Statist. Sci. 17149 (2002).

[2] F. Abe et al., Phys. Rev. Lett. 73(2) 225 (1994).

[3] F. Abe et al., Phys. Rev. Lett. 74(14) 2626 (1995).

[4] J. Neyman and E. S. Pearson, Phil. Trans. R. Soc. A 239289 (1933).

[5] A.Wald, Statistical Decision Functions. New York: Wiley (1950).

[6] L. J. Savage, The Foundations of Statistics. New York: Wiley (1954).

[7] R.A. Fisher, Statistical Methods and Scientific Inference. Edinburgh: Oliver and Boyd (1956).

[8] G.J. Feldman and R.D. Cousins, Phys. Rev. D 573873 (1998).

[9] D.A.S. Fraser, Statist. Sci., to appear (2003).

[10] L.J. Gleser, Statist. Sci. 17161 (2002).

[11] M. Mandelkern, Statist. Sci. 17171 (2002).

[12] R.A. Fisher, Proc. Roy. Soc. A 144, 285 (1934).

[13] D.R. Cox, Ann. Math. Statist. 29, 357 (1958).

[14] C. Giunti, Phys. Rev. D 59113000 (1999).

[15] M. Mandelkern and J. Schultz, J. Math. Phys. 415701 (2000).

[16] S. Ciampolillo, Il Nuovo Cimento 111, 1415 (1998).

[17] B. P. Roe and M.B. Woodroofe, Phys. Rev. D 60053009 (1999).

[18] G. Zech, Nuclear Instruments and Methods A277, 608 (1989).

[19] M.B. Woodroofe and H. Wang, Ann. Statist. 281561 (2000).

[20] R.D. Cousins, Phys. Rev. D 62098301 (2000).

[21] B.P. Roe and M.B. Woodroofe, Phys. Rev. D 63013009 (2001).

[22] L.T. Brown, T.T. Cai and A. DasGupta, Statist. Sci. 16, 101, (2001).

[23] L. Baker, Amer. Statist. 56 85, (2002).

[24] D.A.S. Fraser, Biometrika 90327 (2003).

[25] D.A.S. Fraser, N. Reid, J. Wu, Biometrika 86249 (1999).

[26] O.E. Barndorff-Nielsen and D.R. Cox. Inference and Asymptotics. , Boca-Raton: Chapman \& Hall/CRC.

[27] N. Reid, Ann. Statist., to appear (2003).

available at www.utstat.utoronto.ca/reid/research.

[28] T.A. Severini. Likelihood Methods in Statistics Oxford: Oxford University Press (2000).

[29] I.M. Skovgaard, Scand. J. Statist. 28, 3-32, (2001). 